\title{
Recent advances and current status of gm-Csf as an adjuvant in DNA vaccines for viral diseases
}

\begin{abstract}
Here, I update the recent advances and current status of Granulocyte macrophage colonystimulating factor (GM-CSF), since we have reported for the first time that porcine GMCSF gene in a DNA vaccine formulation exerted immuno-adjuvant and protective effects against Aujeszky's (Pseudorabies)viral disease to the natural host pigs with a single vaccination. GM-CSF has been broadly used as an adjuvant in preclinical DNA vaccine studies for cancer and viral diseases. Currently, GeoVax Labs, Inc. reported a recombinant HIV vaccine (GEO-D03) that co-expresses the human GM-CSF and non-infectious HIV1 virus-like particles (VLPs) is being evaluated in HIV infected young adults in several Phase I studies (NCT01571960). In addition, we summarized here the outcomes of the use of GM-CSF in DNA vaccine for other viral diseases. Further, phase 3 studies reported that GM-CSF showed an improvement in patient outcome when applied in combination with suitable anti-tumor vaccines. However, GM-CSF in excessive levels may expand myeloid suppressor cells that were shown to dampen adaptive immune responses.
\end{abstract}

Keywords: granulocyte macrophage colony-stimulating factor, gm-Csf, genetic adjuvant, DNA vaccine, viral disease, cancer, clinical tria
Volume 2 Issue 3 - 2015

\author{
Chandra Somasundaram \\ University of Houston-Victoria,Texas Biomedical Institute, \\ Missouri City, USA
}

\begin{abstract}
Correspondence: Chandra Somasundaram, University of Houston-Victoria, Texas Biomedical Institute, Missouri City,
\end{abstract} Texas, USA, Email SomasundaramC@uhv.edu

Received: May 31, 2015 | Published: June 16, 2015
Abbreviations: GM-CSF, granulocyte macrophage colonystimulating factor; HIV, human immunodeficiency virus; SIV, simian immunodeficiency virus

\section{Introduction}

Here, I update the recent advances and current status of Granulocyte macrophage colony-stimulating factor (GM-CSF), since we have reported for the first time ${ }^{1,2}$ that a DNA vaccine formulation with porcine GM-CSF gene exerted immuno-adjuvant effects and protected the natural host pigs against Aujeszky's (Pseudorabies) viral disease with single vaccination. The hematopoietic cytokine GM-CSF has been shown as an efficient adjuvant in DNA vaccine preclinical studies for cancer and viral diseases. Xiang $\mathrm{Z}$ et al. ${ }^{3}$ first reported that GM-CSF is a genetic adjuvant for DNA vaccine.

\section{GM-CSF as a genetic adjuvant for HIV DNA vaccine in human clinical trials}

A recombinant HIV vaccine (GEO-D03) that co-expresses the human GM-CSF and non-infectious HIV-1 virus-like particles (VLPs) is being currently evaluated in HIV infected young adults in several Phase I studies (NCT01571960- 2015). ${ }^{4,5}$ This trial will determine whether this vaccine will provide excellent protection in humans as in macaques by simian immunodeficiency virus (SIV)-prototype (NCT01909414-2013). ${ }^{6}$ Lai et al. 2011 reported that the SIV vaccine co-expressing GM-CSF achieved significantly higher reduction in risk of infection and protected more SIV challenged macaques in preclinical studies. In addition, this vaccine elicited both anti-viral T cells and antibody. The vaccine-induced prevention of infection was shown to increase from $25 \%$ to $71 \%$ in the presence of GM-CSF. ${ }^{7}$ The Outcomes of the use of GM-CSF as genetic adjuvant in DNA vaccine for other viral diseases is given in Table 1.

\section{Use of GM-CSF in cancer}

GM-CSF was found the most efficient adjuvant for cancer cell vaccines in early preclinical screens of retroviral-expressed cytokines. ${ }^{27}$ Further, the ability of the fused GM-CSF to elicit antitumor immune responses and boost vaccine efficiency is found in the first licensed cancer vaccine, Provenge. ${ }^{28}$ Despite, a number of studies demonstrating cytokines can act as adjuvants in tumor vaccines, the cost prevent their widespread use, except for the GM-CSF. More recently, GM-CSF has shown improved patient outcome in phase 3 studies when applied in combination with suitable anti-tumor vaccines. ${ }^{29}$ In addition, GM-CSF is licensed to use as an adjuvant in a fusion protein for a dendritic cell therapy for prostate cancer and for recovery and replacement of white blood cells following bone marrow transplantation and chemotherapy. ${ }^{30}$ However, GM-CSF in excessive levels may expand myeloid suppressor cells that were shown to dampen adaptive immune responses. ${ }^{31-33}$

Table I Efficacy and outcomes of GM-CSF as genetic adjuvant in DNA vaccines for viral diseases

\begin{tabular}{ll}
\hline Virus & Efficacy/Outcome of GM-CSF \\
\hline & Pigs immunized with Cap-GM-CSF subunit vaccine showed significantly \\
& higher levels of PCV2-specific antibodies and neutralizing antibodies \\
& and higher average daily weight gain than pigs receiving immunized with \\
the Cap subunit vaccine and a commercial vaccine (Ingelvac CircoFLEX & P $<0.05)$ after wild-type PCV2 challenge.
\end{tabular}

Reference 
Table continued...

\begin{tabular}{ll} 
Virus & Efficacy/Outcome of GM-CSF \\
\hline Flaviviridae Virus & $\begin{array}{l}\text { Reported as complex and diverse, ranging from enhancement to } \\
\text { suppression, depending on the immunogen of Flaviviridae virus DN }\end{array}$
\end{tabular}

Flaviviridae Virus

Simian ImmunoDeficiency Virus

HIVIAIDS

Foot and Mouth Disease

Japanese Encephalitis Virus

HIV

Dengue Virus

Influenza Virus

Hepatitis BVirus

Bronchitis Virus

Feline Immuno Deficiency Virus

Porcine Reproductive and Respiratory Syndrome Virus

Hepatitis CVirus

Simian-Human Immuno Deficiency Virus

HIV-I Gag

Equine Herpes Virus

HIV-I Env

Aujeszky's (Pseudorabies) Viral Disease. suppression, depending on the immunogen of Flaviviridae virus DNA

vaccine candidates.

The co-expressed GM-CSF increased vaccine-induced prevention of infection from $25 \%$ to $71 \%$ in simian immunodeficiency virus in macaques.

GEO-D03, a DNA vaccine that expresses human GM-CSF and noninfectious HIV-I virus-like particles .entered into human trials.

A phase I study of the safety and immunogenicity of DNA/MVA immunizations with co-expressed GM-CSF in HIV-I infected young adults $\quad 5,6$ with suppressed viremia on HAART.

Efficacy of the DNA vaccine with GM-CSF was improved further in reducing the clinical disease and virus excretions by electroporation.

Reported no protection

Induced long-lived humoral and cell mediated immune memory responses.

DVI challenged mice showed long-term IgG response, strong cytotoxic T lymphocyte activity, produced high levels of splenocytesecreted interferon- $\gamma$ and interleukin- 2 and sufficient protection after immunization with pCAG-DVI-GM-CSF immunization than PCAGDVI/E alone

Induced stronger immunogenicity and protection from virus challenge in Aotus monkeys.

GM-CSF gene enhanced systemic and mucosal immunogenicity of the HA DNA vaccine in Rhesus macaque

HBV-S gene fused with GM-CSF strengthened the immune effects of the HBV DNA vaccine in HBV-transgenic mice

pVAX-chGM-CSF and pVAX-SI provided more protection against IBV challenge in chickens than $\mathrm{PVAX}-\mathrm{SI}$ vaccination alone.

Preserved global CD4 T lymphocyte function after the challenge.

Significantly enhanced the humoral and cellular immune responses and protection against PRRSV challenge in pigs

Reported no change in the ThI/Th2 balance as compared with simultaneous IL-23 administration.

Co-immunization with Flt3-L and GM-CSF shown promise in the development of an effective antiviral $\mathrm{HCV}$ vaccine

Enhanced $\lg$ A response was associated with the best protection, but did not achieve significance.

Demonstrated strong antibody and CTL responses and a protective response against infection with recombinant vaccinia virus expressing HIV-I Gag.

DNA vaccine with GM-CSF, formulated in DMRIE-DOPE significantly improved virus neutralizing antibody responses to EHV-I.

The adjuvant treated group showed significantly better control to the challenge than the non-GMCSF group.

9

Bicistronic DNA vaccines containing GM-CSF elicited remarkably potent CD4(+) T cell responses (1) (1)

We demonstrated that the Porcine GM-CSF gene in a DNA vaccine formulation exerted immuno-adjuvant and protective effects with single I vaccination in the natural host pigs against Aujeszky's disease. 


\section{Acknowledgements}

None.

\section{Conflict of interest}

Author declares that there is no conflict of interest.

\section{References}

1. Somasundaram C, Takamatsu H, Andréoni C, et al. Enhanced protective response and immuno-adjuvant effects of porcine GM-CSF on DNA vaccination of pigs against Aujeszky's disease virus. Vet Immunol Immunopathol. 1999;70(3-4):277-287.

2. Dufour V, Chevallier S, Cariolet R, et al. Induction of Porcine Cytokine mRNA Expression after DNA Immunization and Pseudorabies Virus Infection. J Interferon Cytokine Res. 2000;20(10):889-895.

3. Xiang Z, Ertl HC. Manipulation of the immune response to a plasmidencoded viral antigen by coinoculation with plasmids expressing cytokines. Immunity. 1995;2(2):129-135.

4. Hellerstein M, Xu Y, Marino T, et al. Co-expression of HIV-1 viruslike particles and granulocyte-macrophage colony stimulating factor by GEO-D03 DNA vaccine. Hum Vaccin Immunother. 2012;8(11):1654-1658.

5. https://clinicaltrials.gov/ct2/show/NCT01571960

6. https://clinicaltrials.gov/ct2/show/NCT01909414

7. Lai L, Kwa S, Kozlowski PA, et al. Prevention of infection by a granulocyte-macrophage colony-stimulating factor co-expressing DNA/ modified vaccinia Ankara simian immunodeficiency virus vaccine. $J$ Infect Dis. 2011;204(1):164-173.

8. Zhang H, Qian P, Peng B, et al. A novel subunit vaccine co-expressing GM-CSF and PCV2b Cap protein enhances protective immunity against porcine circovirus type 2 in piglets. Vaccine. 2015;33(21):2449-2456.

9. Chen H, Gao N, Wu J, et al. Variable effects of the co-administration of a GM-CSF-expressing plasmid on the immune response to flavivirus DNA vaccines in mice. Immunol Lett. 2014;162(1 Pt A):140-148.

10. Fowler V, Robinson L, Bankowski B, et al. A DNA vaccination regime including protein boost and electroporation protects cattle against footand-mouth disease. Antiviral Res. 2012;94(1):25-34.

11. Chen H, Gao N, Fan D, et al. Suppressive effects on the immune response and protective immunity to JEV DNA vaccine by co-administration of a GM-CSF-expressing plasmid in mice. PLoS One. 2012;7(4):e34602.

12. Mahdavi M, Ebtekar M, Khorram Khorshid HR, et al. ELISPOT analysis of a new CTL based DNA vaccine for HIV-1 using GM-CSF in DNA prime/peptide boost strategy: GM-CSF induced long-lived memory responses. Immunol Lett. 2011;140(1-2):14-20.

13. Zheng Q, Fan D, Gao N, et al. Evaluation of a DNA vaccine candidate expressing prM-E-NS1 antigens of dengue virus serotype 1 with or without granulocyte-macrophage colony-stimulating factor (GM-CSF) in immunogenicity and protection. Vaccine. 2011;29(4):763-771.

14. Raviprakash K, Ewing D, Simmons M, et al. Needle-free Biojector injection of a dengue virus type 1 DNA vaccine with human immunostimulatory sequences and the GM-CSF gene increases immunogenicity and protection from virus challenge in Aotus monkeys. Virology. 2003;315(2):345-352.

15. Loudon PT, Yager EJ, Lynch DT, et al. GM-CSF increases mucosal and systemic immunogenicity of an H1N1 influenza DNA vaccine administered into the epidermis of non-human primates. PloS One. 2010;5(6):e11021

16. Qing Y, Chen M, Zhao J, et al. Construction of an HBV DNA vaccine by fusion of the GM-CSF gene to the HBV-S gene and examination of its immune effects in normal and HBV-transgenic mice. Vaccine. 2010;28(26):4301-4307.
17. Tan B, Wang H, Shang L, et al. Coadministration of chicken GM-CSF with a DNA vaccine expressing infectious bronchitis virus (IBV) S1 glycoprotein enhances the specific immune response and protects against IBV infection. Arch Virol. 2009;154(7):1117-1124.

18. Maksaereekul S, Dubie RA, Shen X, et al. Vaccination with vif-deleted feline immunodeficiency virus provirus, GM-CSF, and TNF-alpha plasmids preserves global CD4 $\mathrm{T}$ lymphocyte function after challenge with FIV. Vaccine. 2009;27(28):3754-3765.

19. Wang X, Li J, Jiang P, et al. GM-CSF fused with GP3 and GP5 of porcine reproductive and respiratory syndrome virus increased the immune responses and protective efficacy against virulent PRRSV challenge. Virus Res. 2009;143(1):24-32.

20. Hartoonian C, Ebtekar M, Soleimanjahi H, et al. Effect of immunological adjuvants: GM-CSF (granulocyte-monocyte colony stimulatingfactor) and IL-23 (interleukin-23) on immune responses generated against hepatitis C virus core DNA vaccine. Cytokine. 2009;46(1):43-50.

21. Encke J, Bernardin J, Geib J, et al. Genetic vaccination with Flt3-L and GM-CSF as adjuvants: Enhancement of cellular and humoral immune responses that results in protective immunity in a murine model of hepatitis C virus infection. World J Gastroenterol. 2006;12(44):7118-7125.

22. Lai L, Vödrös D, Kozlowski PA, et al. GM-CSF DNA: an adjuvant for higher avidity $\mathrm{IgG}$, rectal IgA, and increased protection against the acute phase of a SHIV-89.6P challenge by a DNA/MVA immunodeficiency virus vaccine. Virology. 2007;369(1):153-167.

23. Qiu JT, Chang TC, Lin CT, et al. Novel codon-optimized GM-CSF gene as an adjuvant to enhance the immunity of a DNA vaccine against HIV-1 Gag. Vaccine. 2007;25(2):253-263.

24. Minke JM, Fischer L, Baudu P, et al. Use of DNA and recombinant canarypox viral (ALVAC) vectors for equine herpes virus vaccination. Vet Immunol Immunopathol. 2006;111(1-2):47-57.

25. Robinson HL, Montefiori DC, Villinger F, et al. Studies on GM-CSF DNA as an adjuvant for neutralizing Ab elicited by a DNA/MVA immunodeficiency virus vaccine. Virology. 2006;352(2):285-294.

26. Barouch DH, Santra S, Tenner-Racz K, et al. Potent CD4+ T cell responses elicited by a bicistronic HIV-1 DNA vaccine expressing gp 120 and GM-CSF. J Immunol. 2002;168(2):562-568.

27. Dranoff G, Jaffee E, Lazenby A, et al. Vaccination with irradiated tumor cells engineered to secrete murine granulocyte-macrophage colonystimulating factor stimulates potent, specific, and long-lasting anti-tumor immunity. Proc Natl Acad Sci. 1993;90:3539-3543.

28. Small EJ, Fratesi P, Reese DM, et al. Immunotherapy of hormonerefractory prostate cancer with antigen-loaded dendritic cells. J Clin Oncol. 2000;18(23):3894-3903.

29. Kaufman HL, Ruby CE, Hughes T, et al. Current status of granulocytemacrophage colony-stimulating factor in the immunotherapy of melanoma. J Immunother Cancer. 2014;2:11.

30. Borrello I, Pardoll D. GM-CSF-based cellular vaccines: a review of the clinical experience. Cytokine Growth Factor Rev. 2002;13(2):185-193.

31. Serafini P, Carbley R, Noonan KA, et al. High-dose granulocytemacrophage colony-stimulating factor-producing vaccines impair the immune response through the recruitment of myeloid suppressor cells. Cancer Res. 2004;64:6337-6343.

32. Filipazzi P, Valenti R, Huber V, et al. Identification of a new subset of myeloid suppressor cells in peripheral blood of melanoma patients with modulation by a granulocyte-macrophage colony-stimulation factorbased antitumor vaccine. J Clin Oncol. 2007;25(18):2546-2553.

33. Parmiani G, Castelli C, Pilla L, et al. Opposite immune functions of GMCSF administered as vaccine adjuvant in cancer patients. Ann Oncol. 2007;18(2):226-232. 